\title{
Nanogel Formation from Dilute Solutions of Clickable Elastin-Like Recombinamers and its Dependence on Temperature: Two Fractal Gelation Modes.
}

\author{
Israel González de Torre*, Luis Quintanilla, Guillermo Pinedo-Martín, Matilde Alonso, José Carlos \\ Rodríguez-Cabello. \\ BIOFORGE, CIBER-BBN, Campus "Miguel Delibes" Centro I+D, Universidad de Valladolid, Paseo Belén 11, 47011, Va- \\ lladolid, Spain.
}

Elastin-like recombinamers, click chemistry, hydrogels, nanogels, microrheology.

\begin{abstract}
Diluted, complementary, click-reactive elastin-like recombinamer (ELR) solutions have been prepared and mixed at two different temperatures, one below and one above the characteristic transition temperature $\left(\mathrm{T}_{\mathrm{t}}\right)$ of these chemically modified ELRs. Size, aspect ratio, zeta potential and microrheological measurements have been carried out on the nanostructures formed under these dilute conditions as a way to better understand the relationship between the final macroscopic properties of ELR-based hydrogels and the molecular conditions governing the initial stages of the chemical crosslinking process that occurs, especially its dependence on the preparation temperature relative to $T_{t}$ As a result, two different fractal modes of gel formation have been found at the two temperatures studied (above and below $T_{t}$ ). Thus, when the reaction mixture is prepared below $T_{t}$, essentially onedimensional linear nanogels with a high aspect ratio are obtained. In contrast, $3 \mathrm{D}$ nanogels are formed above $\mathrm{T}_{\mathrm{t}}$, with spherical shapes predominating. These different structures seem to reflect the two molecular organizations of the single components of the mixture under these conditions, namely extended chains below $\mathrm{T}_{\mathrm{t}}$ and a globular arrangement above $\mathrm{T}_{\mathrm{t}}$. In addition to the interest in these nanogels as models for understanding the formation of microscopic structures and differential macroscopic properties under more conventional hydrogel-formation conditions, these nanogels are of interest due to their thermoresponsiveness and biocompatibility, which provide them with potential uses for drug delivery and other biomedical applications in living systems.
\end{abstract}

\section{INTRODUCTION}

New materials, many of which are intended for use as gels, especially in the bioscience fields, are continuously coming to the fore.

Elastin-like recombinamers (ELRs) are proteinaceous biomaterials based on the repetition of certain sequences from natural elastin ${ }^{1,2}$. These compounds are designed and produced by recombinant DNA technology, thus allowing complete control of their structure and amino acid sequence. ELRs can include different bioactive sequences3, such as those governing protease sensitivity or cell adhesion, etc. These ELRs exhibit cell friendly behavior, tunable mechanical properties, thermal sensitivity and an ability to self-assemble ${ }^{3,4}$. All these properties are interesting for the most cutting-edge applications in the fields of nanotechnology and biomedicine. Their thermal sensitivity is characterized by a critical temperature in aqueous solution, known as the transition temperature $\left(T_{t}\right)$, which is associated with a conformational reorganization at the molecular level. Thus, whereas the polymer chains are soluble in water below $T_{t}$, they self-assemble into nano- and microaggregates above this temperature, becoming insoluble. This process is completely reversible.

We have recently, designed, synthesized, and bioproduced elastin-like recombinamer catalyst-free click gels (ELR-
CFCGs), the characterization of which has been reported in detail elsewhere 5 . The temperature dependence of these gels exhibits two different mechanical behaviors, thereby mimicking the thermosensitive behavior of the single ELRs that form the hydrogel. This dual behavior suggested to us the presence of two different processes during gel formation depending on whether hydrogel formation was carried out above or below the $T_{t}$ of each component. In particular, RGD-azide and VKVx24-cyclooctyne $\left(T_{t}\right.$ of 21 and $15^{\circ} \mathrm{C}$, respectively ${ }^{5}$ ) have been used in this work.

Although hydrogels have been widely studied in the literature, our understanding of the early stages of gel formation requires further studies in order to gain a better insight into the relationship between the final properties of the hydrogel and the conditions present during the early stages of the crosslinking reaction, when the initial gel seeds start to appear. These initial stages are equivalent to the conditions existing under dilute conditions where, because of the low quantity of reagent mass, the hydrogels formed are unable to grow above the nano-aggregate level and are therefore models for the initial emerging structures found under more conventional conditions. In addition, these entities are interesting in and of themselves. Some recent studies have been devoted to the topic of nano- and microgels ${ }^{6-8}$. 
Microrheology involves the use of several techniques to study the rheological properties of solutions, nanoparticles and, in general, soft materials by studying the motion of colloidal probes dispersed within the samples ${ }^{9-12}$. Several experimental methods for measuring the dynamics of those probes have been proposed in the literature ${ }^{12}$. However, whereas active microrheology involves external forces acting on the microparticles used as probes (tracers), passive microrheology make use of the thermal (Brownian) motion of these tracers. In addition to early microrheological measurements on crosslinked polymer systems ${ }^{13-15}$, the microrheology of cross-linked polyacrylamide networks ${ }^{16}$, poly(vinyl alcohol) hydrogels ${ }^{17}$, microtubule networks ${ }^{18}$, and F-actin networks ${ }^{19-21}$ has been reported in the literature.

In this work, the size and surface charge (zeta potential) of the nanogels emerging from dilute click-chemistry crosslinking ELR solutions have been correlated with the viscoelastic properties of these solutions obtained using passive microrheology.

\section{MATERIAL AND METHODS}

\section{Sample preparation}

Details regarding the compositions of the ELRs used in this work, as well as the chemical modifications used to obtain their clickable derivatives (ELR-Azide and ELR-cyclooctyne), can be found elsewhere ${ }^{5}$. The range of concentrations analyzed in this work was chosen to represent dilute solutions far from the sol-gel transition. As such, the samples are homogeneous and ergodic assumptions remain valid ${ }^{16}$. Three different concentrations $(0.1,0.5$, and $1 \mathrm{mg} / \mathrm{mL})$ of each modified ELR were prepared, filtered (using a $0.45 \mu \mathrm{m}$ PVDF syringe filter) and kept overnight at $4^{\circ} \mathrm{C}$. They were then mixed (at 4 or $37^{\circ} \mathrm{C}$ ) to obtain the desired concentration of click solutions. These solutions were maintained at 4 or $37^{\circ} \mathrm{C}$ for 24 hours. A $\mathrm{pH}$ of 7.1 was achieved when both components were mixed at the appropriate concentrations in $\mathrm{mQ}$ water.

\section{Experimental techniques}

Dynamic light scattering, Zeta potential, and microrheology measurements were performed using a Zetasizer Nano ZSP (Malvern Instruments) equipped with a $10 \mathrm{~mW} \mathrm{He}-\mathrm{Ne}$ laser at a wavelength of $633 \mathrm{~nm}$.

Dynamic Light Scattering. For DLS measurements, samples were introduced into polystyrene cuvettes and stabilized for 5 min at the desired temperature of 4 or $37^{\circ} \mathrm{C}$.

The DLS autocorrelation functions were measured at 4 and $37^{\circ} \mathrm{C}$ for all three concentrations. The autocorrelation functions were used to obtain the size distribution and polydispersity index. DLS measurements were performed using a noninvasive back-scattering ${ }^{22}$ configuration.

Zeta Potential. The $\zeta$ potential of the solutions was monitored at 4 and $37^{\circ} \mathrm{C}$. The $\zeta$-potential values, which were determined using the Smolukowski equation relating ionic mobility to surface charge, were obtained as the average of 10 repeated measurements. $\zeta$ measurements were performed using phase analysis light scattering ${ }^{23}$.

Cryo-Transmission Electron Microscopy (Cryo-TEM). Cryo-TEM measurements were accomplished using a JEOL JEM-FS2200 electron microscope operating at an accelerating voltage of $200 \mathrm{kV}$. Stabilized click solutions at $4{ }^{\circ} \mathrm{C}$ and $37^{\circ} \mathrm{C}$ for several concentrations were placed on a preheated plasmatreated carbon-coated copper grid, followed by cryovitrifica- tion of the sample using a Gatan Cryoplunge CP3. A zero-loss mode was used to improve image contrast.

\section{Microrheology.}

Passive microrheology was based on Dynamic Light Scattering with improved single scattering detection ${ }^{22}$.

Prior to the microrheology measurements, a careful selection of the tracer has to be made ${ }^{9,24}$. Two different tracer particles for DLS-based microrheology were considered in this work: surface-sulfonated polystyrene spheres with a mean diameter of $1101 \mathrm{~nm} \pm 23 \mathrm{~nm}$, and surface-carboxylated melamine resin particles with a mean diameter of $1020 \mathrm{~nm} \pm 40 \mathrm{~nm}$.

Polystyrene tracer particles were purchased from Thermo Scientific (catalog number 4011A, nominal diameter $1.1 \mu \mathrm{m}$, density $=1.05 \mathrm{~g} / \mathrm{cm}^{3}$ ). Surface-carboxylated melamine resin particles were purchased from microParticles $\mathrm{GmbH}$ (catalog number MF-COOH-particles, nominal diameter $1.0 \mu \mathrm{m}$, density $\left.=1.51 \mathrm{~g} / \mathrm{cm}^{3}\right)$.

The tracer particles were added to the click solutions and allowed to fully disperse for at least $12 \mathrm{~h}$ prior to taking any measurement. The particle concentration for the microrheological measurements was selected to ensure that the lightscattering process was dominated by the particles, thus meaning that a single, narrow peak was observed in the lightscattering experiment. For each experiment, $10 \mu \mathrm{L}$ of tracer was added to $500 \mu \mathrm{L}$ of sample $\mathrm{e}^{25}$.

\section{RESULTS AND DISCUSSION}

DLS and Zeta-potential measurements, and cryo-TEM micrographs

Size-distribution measurements for each single modified ELR (RGD-azide and VKVx24-cyclooctyne) were carried out at both temperatures, and no stable structures were found (PDI close to 1). The same measurements for the nanogels present in our clickable solutions were performed by DLS below and above the $T_{t}$. Bimodal size distributions were found at both temperatures (Fig. 1), with a mean structure hydrodynamic diameter of 50 and $400 \mathrm{~nm}$ for the two lowest concentrations. These values are slightly higher $(100$ and $500 \mathrm{~nm})$ for the 1 $\mathrm{mg} / \mathrm{mL}$ solution at both temperatures. As can be seen from Fig. 1 , at $4{ }^{\circ} \mathrm{C}$ the majority of the nanogel population has a size distribution of less than than $100 \mathrm{~nm}$. This trend is opposite at $37^{\circ} \mathrm{C}$. However, these hydrodynamic diameters must be taken with caution as the polydispersity value is high $(0.325$ and 0.300 at 4 and $37^{\circ} \mathrm{C}$, respectively) and DLS is less accurate under these conditions. 
A)

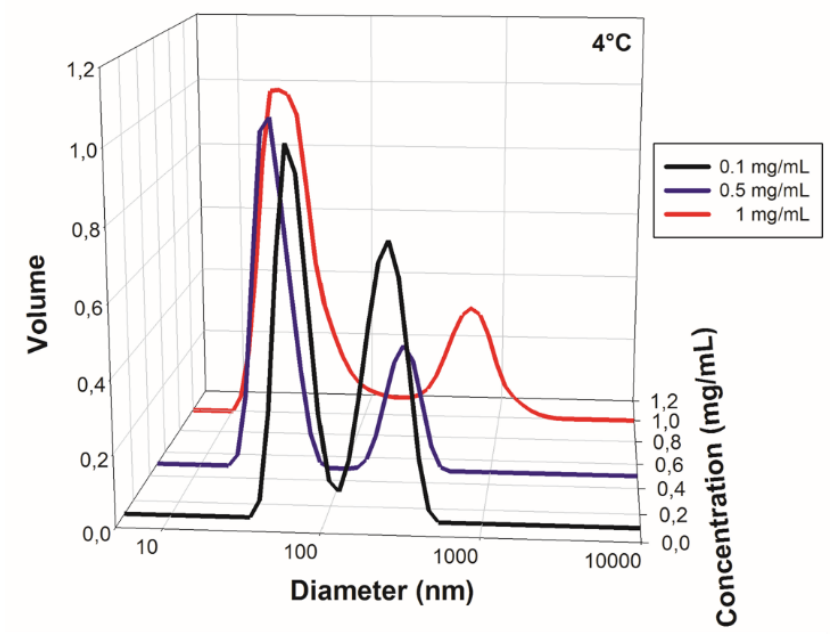

B)

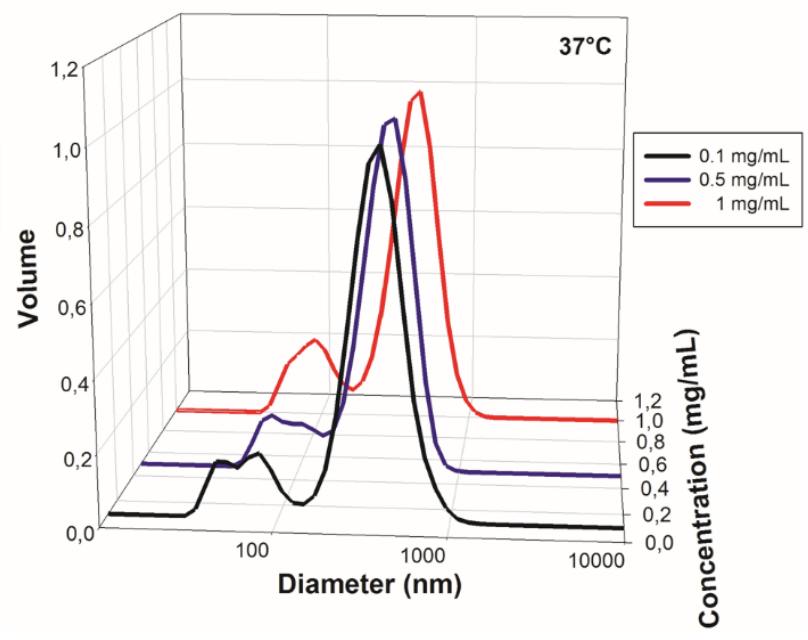

Fig. 1. Normalized size distribution as a function of polymer concentration at 4 (A) and $37^{\circ} \mathrm{C}(\mathrm{B})$. The ratio of the volume to the peak volume of each measurement is used.

The size and shape of the nanogels were further evaluated by cryo-TEM. As can be seen in Fig. 2, the observed structures are completely different when obtained below (Fig. 2A) and above (Fig. 2B) their $\mathrm{T}_{\mathrm{t}}$. Thus, whereas the structures adopt an anisotropic geometry with a quasi-linear shape at $4^{\circ} \mathrm{C}$, these structures adopt a spherical shape at $37^{\circ} \mathrm{C}$. As can be seen from Figure 2, the size of the observed structures is in good agreement with the size distribution obtained by DLS.

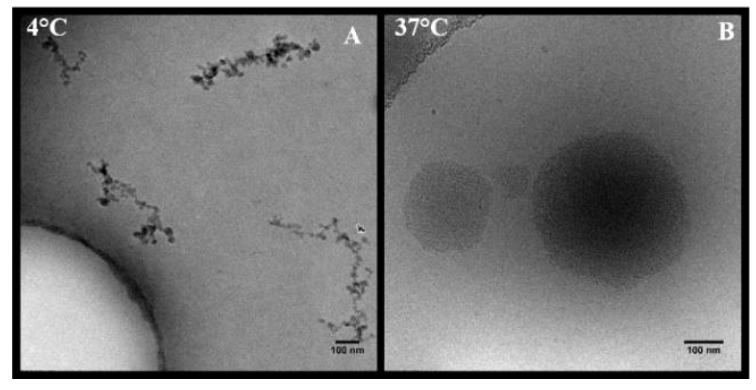

Fig. 2. Cryo-TEM micrographs of click nano-gels at 4 (A) and $37^{\circ} \mathrm{C}(\mathrm{B})$.

Zeta-potential measurements (Fig. 3) also revealed two different trends in the distribution of the effective surface charge. The charge in these ELRs is mainly derived from the positively charged lysine side chains (epsilon amine group), which are not modified during cyclooctyne or azide addition. Thus, whereas a constant value of about $+26 \mathrm{mV}$ was found for all concentrations at $37^{\circ} \mathrm{C}$, this value decreased from +15 to +6 $\mathrm{mV}$ with concentration at $4^{\circ} \mathrm{C}$. According to the literature ${ }^{26-29}$, these values suggest the presence of nano-gels with high and low stability at 37 and at $4^{\circ} \mathrm{C}$, respectively.

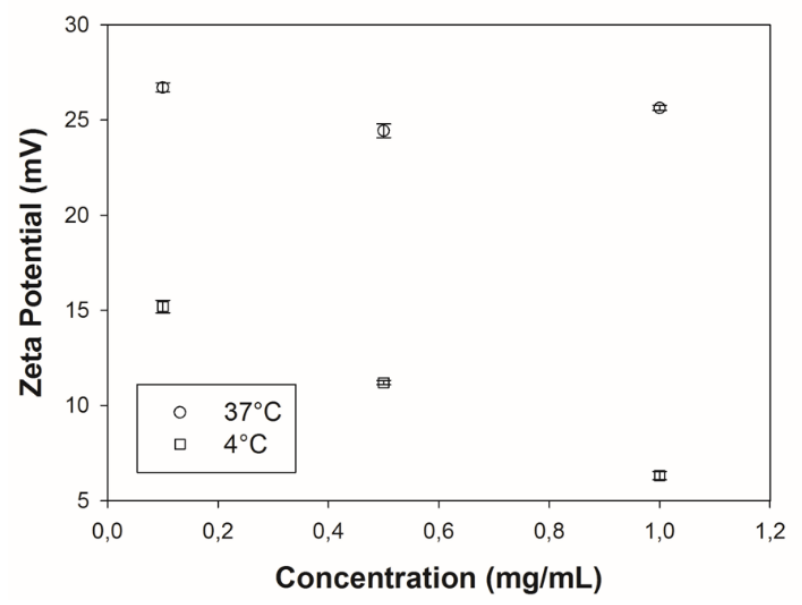

Fig. 3. Zeta potential $(\zeta)$ for the nanogels as a function of recombinamer concentration.

\section{Microrheology measurements}

To test the bulk response of the click-reactive ELR solution, the assumption of continuum viscoelasticity must be satisfied. As a result, the probe size has to be larger than the relevant length scale of the nanogels. Moreover, a minimization of tracer-matrix interactions, the extent of which is estimated by zeta potential measurements, is required.

In order to select the best candidate as a tracer for this system, tracer particles of two different compositions, namely polystyrene and melamine, were tested by adding them to the click-reactive ELR solution. This selection was made on the basis of the degree of interaction between the ELR gels and the tracer. The tracers were added to the same concentration as the ELR concentration and the zeta potential was then measured at $37^{\circ} \mathrm{C}$ for the solutions with tracer. Polystyrene particles gave rise to a $30-35 \%$ change compared to the previous zeta potential for the click-reactive ELR solution obtained without tracer, whereas only a 5-7\% change was found for melamine particles. These findings are in agreement with literature data 
for similar systems ${ }^{19,25}$. As a result, melamine resin particles were used for the microrheological measurements in this work.

In a microrheological experiment, the mean square displacement (MSD) provides a quantitative insight into the thermal motion of racer particles. This MSD is obtained from the autocorrelation function when DLS is used to follow the tracer.

A)

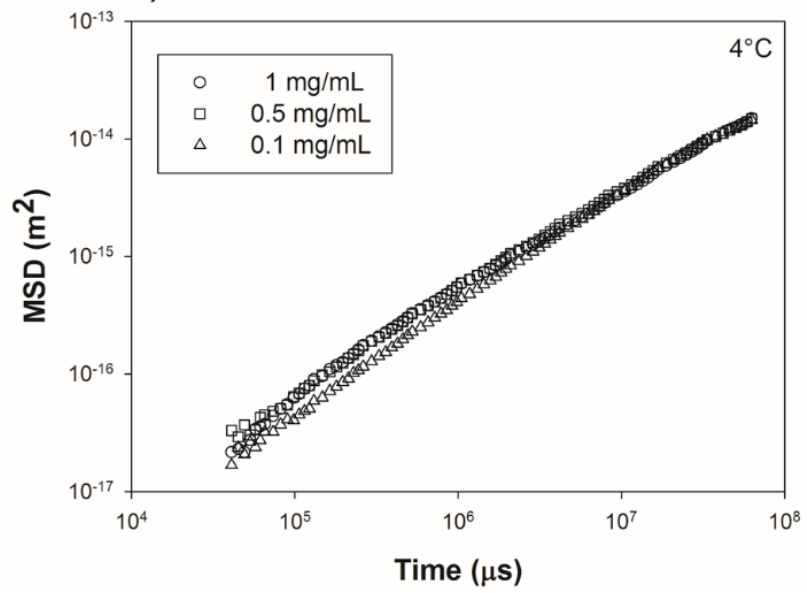

B)

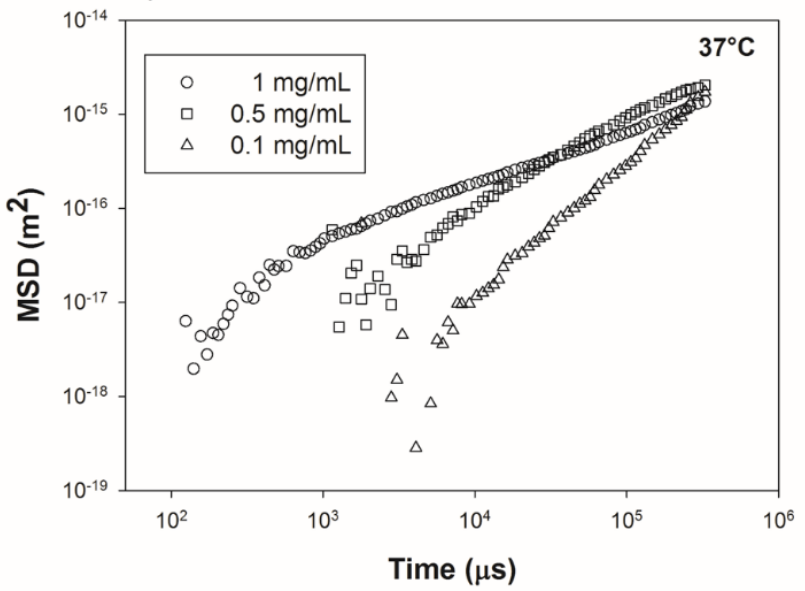

Fig. 4. Mean square displacement (MSD) of the tracer particles for several recombinamer concentrations at 4 (A) and $37^{\circ} \mathrm{C}(\mathrm{B})$. Each curve corresponds to the average of three different measurements.

In Fig. 4, the MSD for the three concentrations considered has been plotted as a function of time at 4 and $37^{\circ} \mathrm{C}$. As can be seen from this figure, a linear response was found at both temperatures on a log-log scale. Relatively similar slopes (close to 1) are observed for the three recombinamer concentrations studied at $4^{\circ} \mathrm{C}$, thus indicating a purely diffusive motion for the tracer. However, at $37^{\circ} \mathrm{C}$ the slope decreases as the concentration increases. According to the literature, this corresponds to a subdiffusive behavior ${ }^{25}$. The motion of the tracer particles is hampered by the slight increase in the size of the nanogels at $1 \mathrm{mg} / \mathrm{mL}$ and, as a result, the MSD slope decreases, thus reflecting an increase in the magnitude of their complex modulus ${ }^{30}$.

A)

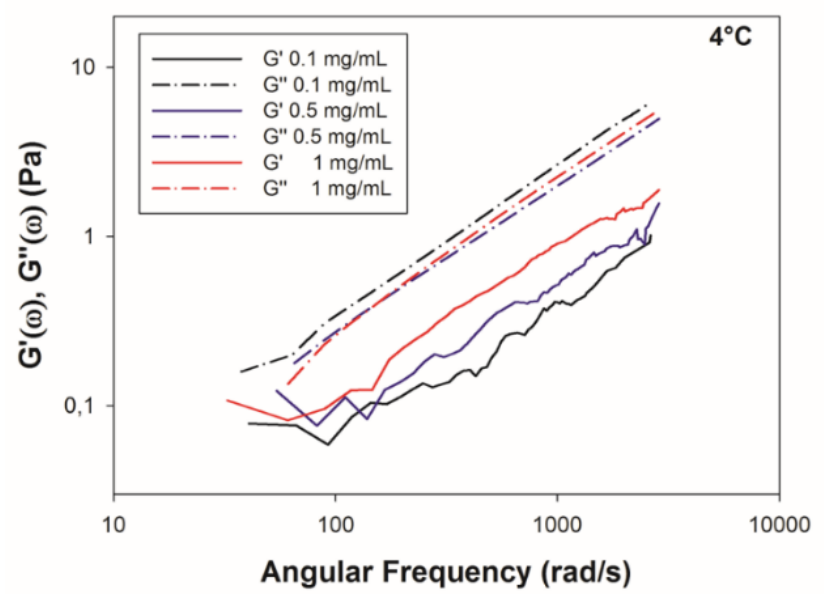

B)

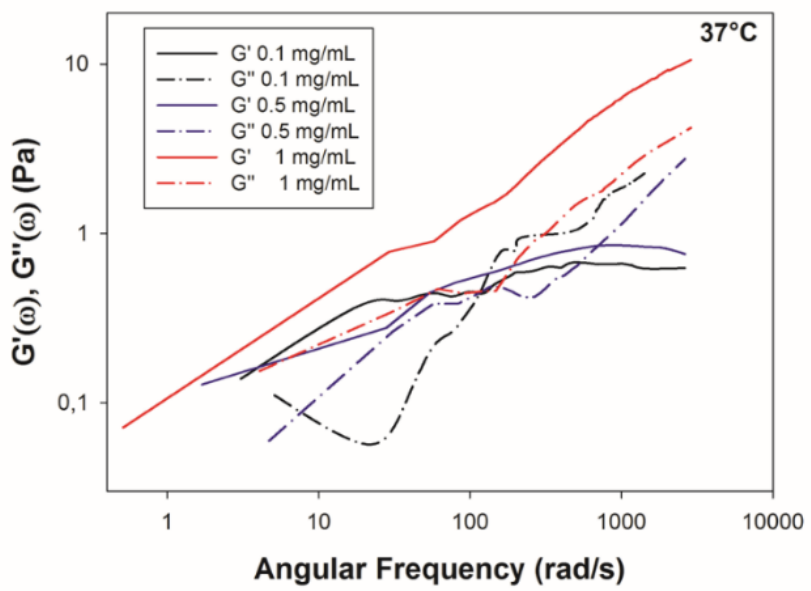

Fig. 5. Storage $\left(\mathrm{G}^{\prime}\right)$ and loss $\left(\mathrm{G}^{\prime \prime}\right)$ moduli as a function of angular frequency at $4(\mathrm{~A})$ and $37^{\circ} \mathrm{C}(\mathrm{B})$ for the studied concentrations $(0.1,0.5$ and $1 \mathrm{mg} / \mathrm{mL}$ ). Each curve corresponds to the average of three different samples. 
Using the generalized Stokes-Einstein relationship ${ }^{9,24}$, the viscoelastic moduli were calculated using the method described by Mason ${ }^{24}$. The dependence of the storage $\left(\mathrm{G}^{\prime}\right)$ and loss $\left(\mathrm{G}^{\prime \prime}\right)$ moduli on angular frequency has been plotted in Fig. 5. $G^{\prime}$ and $G^{\prime \prime}$ were frequency-dependent in the range considered at both temperatures. At $4^{\circ} \mathrm{C}, \mathrm{G}^{\prime \prime}$ is higher than $\mathrm{G}^{\prime}$, thus indicating that the sample is a viscoelastic fluid over the entire range of frequencies considered. No significant changes were found for $\mathrm{G}^{\prime \prime}$ with recombinamer concentration, although $\mathrm{G}^{\prime}$ increased slightly with concentration at frequencies higher than $200 \mathrm{rad} / \mathrm{s}$. At $37^{\circ} \mathrm{C}$, similar $\mathrm{G}^{\prime}$ values were obtained for the concentrations of 0.1 and $0.5 \mathrm{mg} / \mathrm{mL}$, and a plateau seems to be reached at around $1000 \mathrm{rad} / \mathrm{s}$. Furthermore, for each concentration, $\mathrm{G}^{\prime}$ dominates over $\mathrm{G}^{\prime \prime}$ up to a frequency of around 100 and $900 \mathrm{rad} / \mathrm{s}$, respectively, at which point a crossover occurs. In contrast, $\mathrm{G}^{\prime}$ clearly dominates over $\mathrm{G}^{\prime \prime}$ for a concentration of $1 \mathrm{mg} / \mathrm{mL}$, and no crossover is found in the frequency range analyzed. $\mathrm{G}^{\prime}$ is higher for this latter concentration than for lower concentrations, and no plateau is observed.

A schematic representation of the proposed mechanism governing the formation and thermal behavior of these nanogels is summarized in Fig. 6. ELRs show a lower critical solution temperature (LCST) behavior in which the ELR molecule is water soluble below $T_{t}$, with hydrophobic hydration being the characteristic and predominant hydration mode. The clathrate-like structure of water molecules surrounding the apolar ELR moieties, which is characteristic of hydrophobic hydration, gives rise to a relatively extended chain with no conformational order ${ }^{31}$. The ELR chains do not assemble under these conditions and remain extended in solution, in this particular case with positively charged lysine residues distributed homogeneously along the backbone. In contrast, this hydrophobic hydration no longer exists at $37^{\circ} \mathrm{C}$, above their $\mathrm{T}_{t}$, and the ELR chains undergo a self-aggregation process dominated by an extensive number of hydrophobic associations and segregation of those aggregated domains from solution. For the particular amphiphilic compositions of the ELRs employed herein, in which apolar domains coexist with polar ones, the resulting molecular arrangement is globular, with the apolar domains forming a core in an attempt to hide from the aqueous environment, with the polar domains (charged lysines) exposed in the shell.

As the LCST behavior of ELRs is an intrinsic property, the tendency shown by the isolated chains prior to cross-linking must still be present and preserved in the cross-linked state. Below $T_{t}$, the cross-linked ELR chain tends to be hydrated and extended, whereas above $T_{t}$ the cross-linked chain shows a folded globular state with extensive inter- and intrachain hydrophobic contacts. These two molecular arrangements appear to govern the way in which the newly formed gel structures grow. Thus, below $T_{t}$ the nanogels adopt a linear structure, which is coincident with the main structure at a molecular level and a certain degree of self-orientation driven by the formation of cross-linking reactions in these extended chains in a zipper-like manner, whereas at temperatures above $T_{t}$ the newly formed gel nanoparticles show a spherical geometry following the globular organization at the molecular level. As such, the geometry observed at the nanometric level appears to be a fractal image of that observed at the molecular level.

The observed zeta potential values are also in agreement with this general description. Thus, below $T_{t}$ the positively charged lysines are not segregated from the rest of the molecular domains but remain relatively evenly distributed, with no particular preference for being in the core or the shell of the newly formed nanogels. As such, the zeta potential is relatively low and decreases as the concentration increases due to the fact that the proportion of lysines exposed on the outer surface of the nanogels with respect to the total number of lysines within the nanogel decreases. In contrast, the nanogels obtained at a temperature above $\mathrm{T}_{\mathrm{t}}$ are dominated by segregation of the apolar and polar domains. At the water-gel interphase, the latter would clearly tend to be exposed towards the water side, thus increasing the zeta potential and making it practically independent of the concentration.

This differential behavior is also supported by the microrheology measurements. Below $T_{t}$, the complex viscoelastic modulus is dominated by the loss modulus ( $\left.G^{\prime \prime}\right)$. As such, the mechanical properties of these nanogels are related to those of a viscoelastic fluid, a state which is compatible with the existence of a number of ELR chains that are fully hydrated and loose, with very few intermolecular interactions other than the crosslinks that give coherence to the nanogel. The mechanical properties would be affected by water diffusing in and out the hydrogel, which results in deformation and explains the high contribution of the loss modulus. However, above $T_{t}$, the chains experience a high number of intra- and intermolecular hydrophobic contacts, which result in consolidation of the solid continuous phase and the emergence of a more purely elastic behavior, where G' clearly dominates over G'.

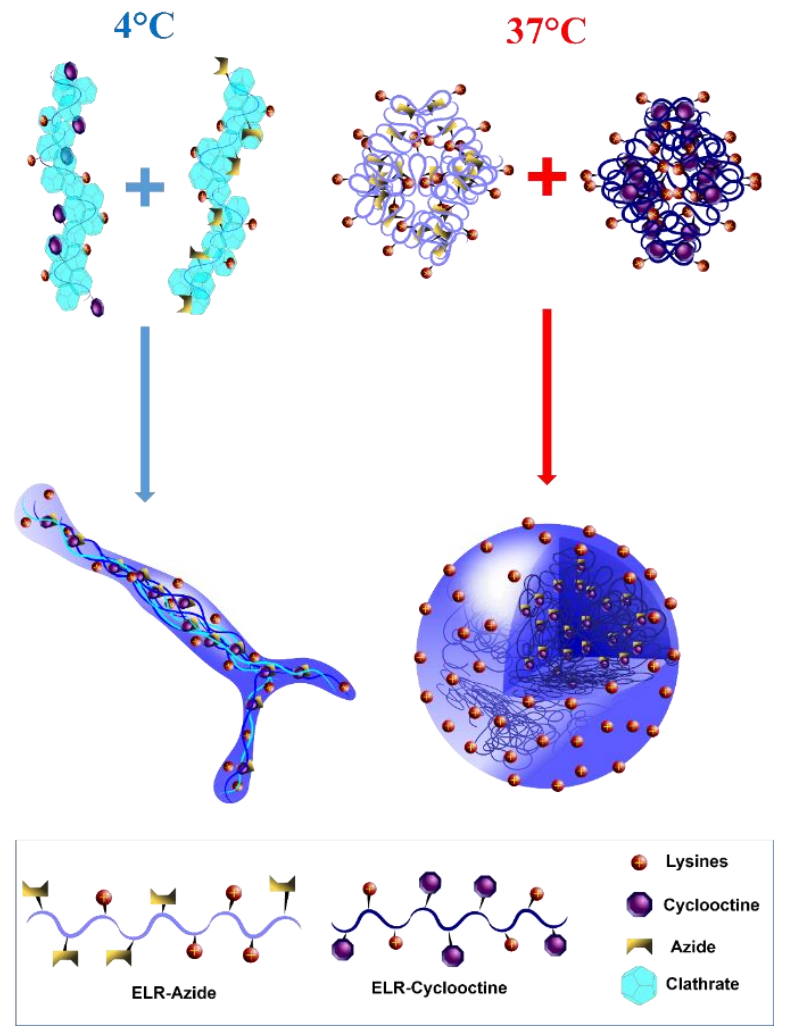

Fig. 6. Schematic representation of the proposed mechanism governing click nanogel formation and the behavior above and below $\mathrm{T}_{\mathrm{t}}$.

\section{CONCLUSIONS}


DLS, zeta potential, and microrheological measurements have been correlated in this work to gain an insight into the influence of temperature, and the concomitant changes in the aggregation state of ELRs around their $T_{t}$, on the microscopic structure and shape of hydrogel formation.

Briefly, for the thermally sensitive ELRs studied herein, the reaction temperature dramatically affects the dimensionality of gel growth for these cross-linked hydrogels. Below $\mathrm{T}_{t}$, gels grow in an essentially monodimensional mode and linear (fibrilar) structures with a high aspect ratio are the dominant geometric feature observed. However, above $T_{t}$, the crosslinking reaction is dominated by the previous self-organization of the ELR chains and their tendency to adopt globular structures. This circumstance seems to drive the newly formed nanogels to achieve a homogeneous, isotropic and threedimensional hydrogel that is spherical in shape. As such, the observed nanometric shapes correspond to the fractal image of the arrangement of the ELR chains below and above $T_{t}$.

These results show, for the first time, that it is possible to easily obtain nanogels with strikingly different topographies. Such nanogels have potential uses in numerous different biomedical uses. Secondly, these results are also relevant as regards our understanding of the different macroscopic properties observed when preparing cross-linked ELR macrogels. According to the results presented here, the crosslinking temperature markedly affects the microstructure of the final hydrogels, with the ELR $T_{t}$ being the characteristic temperature that determines one mode of gelation or another.

\section{AUTHOR INFORMATION}

\section{Corresponding Author}

*Israel González de Torre. igonzalez@bioforge.uva.es BIOFORGE, Campus "Miguel Delibes" Centro I+D, CIBERBBN, Universidad de Valladolid, Paseo Belén 11, 47011, Valladolid, Spain.

\section{ACKNOWLEDGMENT}

We acknowledge financial support from the EU through the European regional development fund (ERDF) NMP3-LA-2011263363, HEALTH-F4-2011-278557, PITN-GA-2012-317304), from the MINECO (MAT-2010-15982, MAT2010-15310, PRIPIBAR-2011-1403 and MAT2012-38043-C02-01), the JCyL (projects VA152A12-2, VA244U13 and VA155A12-2), the CIBER-BBN, and the JCyL and the Instituto de Salud Carlos III under the "Network Center of Regenerative Medicine and Cellular Therapy

\section{REFERENCES}

1. Urry, D. W.; Urry, K. D.; Szaflarski, W.; Nowicki, M., Elastic-Contractile Model Proteins: Physical Chemistry, Protein Function and Drug Design and Delivery. Adv. Drug Delivery Rev. 2010, 62 (15), 1404-1455.

2. Karle, I. L.; Urry, D. W., Crystal Structure of Cyclic (APGVGV)2, an Analog Of Elastin, And A Suggested Mechanism
For Elongation/Contraction Of The Molecule. Biopolymers 2005, 77

(4), 198-204.

3. Rodríguez-Cabello, J. C.; Martín, L.; Alonso, M.; Arias, F.

J.; Testera, A. M., "Recombinamers" as Advanced Materials for the Post-Oil Age. Polymer 2009, 50 (22), 5159-5169.

4. Wright, E. R.; Conticello, V. P., Self-Assembly of Block Copolymers Derived from Elastin-Mimetic Polypeptide Sequences. Adv. Drug Delivery Rev 2002, 54 (8), 1057-1073.

5. González de Torre, I.; Santos, M.; Quintanilla, L.; Testera, A.; Alonso, M.; Rodríguez Cabello, J. C., Elastin-Like Recombinamer Catalyst-Free Click Gels: Characterization of Poroelastic and Intrinsic Viscoelastic Properties. Acta Biomater 2014, 10 (6), 2495-2505.

6. Jiang, Y.; Chen, J.; Deng, C.; Suuronen, E.; Zhong, Z., Click Hydrogels, Microgels and Nanogels: Emerging Platforms for Drug Delivery and Tissue Engineering. Biomaterials 2014, 35 (18), 4969-4985.

7. Steinhilber, D.; Witting, M.; Zhang, X.; Staegemann, M.; Paulus, F.; Friess, W.; Küchler, S.; Haag, R., Surfactant Free Preparation of Biodegradable Dendritic Polyglycerol Nanogels by Inverse Nanoprecipitation for Encapsulation and Release of Pharmaceutical Biomacromolecules. . J. Controlled Release. 2013, 169 (3), 289-295.

8. Dmitri, O.; Sujit, K.; Zheyi, Y.; Xia, Y.; Jöns, H., Orthogonal Chemoselective Assembly of Hyaluronic Acid Networks and Nanogels for Drug Delivery. Macromolecules 2013, 46.

9. Mason, T. G.; Weitz, D. A., Optical Measurements of Frequency-Dependent Linear Viscoelastic Moduli of Complex Fluids. Phys. Rev. Lett. 1995, 74 (7), 1250-1253.

10. Cicuta, P.; Donald, A. M., Microrheology: A Review of the Method and Applications. Soft Matter 2007, 3 (12), 1449-1455.

11. Breedveld, V.; Pine, D. J., Microrheology as a Tool for High-Throughput Screening. J. Mater. Sci. 2003, 38 (22), 4461-4470.

12. Squires, T.; Mason, T., Fluid Mechanics of Microrheology. Annu. Rev. Fluid Mech. 2009. 640, 343.

13. Schnurr, B.; Gittes, F.; MacKintosh, F. C.; Schmidt, C. F., Determining Microscopic Viscoelasticity in Flexible and Semiflexible 
Polymer Networks from Thermal Fluctuations. Macromolecules 1997, 30 (25), 7781-7792.

14. Narita, T.; Knaebel, A.; Munch, J.-P.; Candau, S. J., Microrheology of Poly(vinyl alcohol) Aqueous Solutions and Chemically Cross-Linked Gels. Macromolecules 2001, 34 (23), 8224-8231.

15. Nisato, G.; Hébraud, P.; Munch, J. P.; Candau, S. J., Diffusing-wave-spectroscopy Investigation of Latex Particle Motion in Polymer Gels. J. Physical Review E 2000, 61 (3), 2879-2887.

16. Dasgupta, B.; Weitz, D., Microrheology of Cross-Linked Polyacrylamide Networks. Phys. Rev. E: Stat., Nonlinear, Soft Matter Phys.2005, 71 (2 Pt 1), 21504.

17. Narita, T.; Mayumi, K.; Ducouret, G.; Hébraud, P., Viscoelastic Properties of Poly(vinyl alcohol) Hydrogels Having Permanent and Transient Cross-Links Studied by Microrheology, Classical Rheometry, and Dynamic Light Scattering. Macromolecules 2013, 46 (10), 4174-4183.

18. Yang, Y.; Bai, M.; Klug, W. S.; Levine, A. J.; Valentine, M. T., Microrheology of Highly Crosslinked Microtubule Networks is Dominated by Force-Induced Crosslinker Unbinding. Soft Matter 2013, 9 (2), 383-393.

19. McGrath, J.; Hartwig, J.; Kuo, S., The Mechanics of FActin Microenvironments Depend on the Chemistry of Probing Surfaces. Biophys. J. 2000, 79 (6), 3258-3266.

20. Lee, H.; Ferrer, J.; Nakamura, F.; Lang, M.; Kamm, R., Passive and Active Microrheology for Cross-Linked F-Actin Networks in vitro. Acta Biomater. 2010, 6 (4), 1207-1218.

21. Luan, Y.; Lieleg, O.; Wagner, B.; Bausch, A., Micro and Macrorheological Properties of Isotropically Cross-Linked Actin Networks. Biophys. J. 2008. 15;94(2):688-93

22. Peters, R., Fiber Optic Device for Detecting the Scattered Light or Fluorescent Light from a Suspension. Google Patents 2000.

23. John, F. M.; Klaus, S.; Brian, V., The Determination of Very Small Electrophoretic Mobilities in Polar and Nonpolar Colloidal Dispersions Using Phase Analysis Light Scattering. J. Colloid Interface Sci.1991, 143.
24. Mason, T. G., Estimating the Viscoelastic Moduli of Complex Fluids Using the Generalized Stokes-Einstein Equation. Rheol. Acta 2000, 39 (4), 371-378.

25. Amin, S.; Rega, C.; Jankevics, H., Detection of Viscoelasticity in Aggregating Dilute Protein Solutions through Dynamic Light Scattering-Based Optical Microrheology. Rheol. Acta 2012, 51 (4), $329-342$.

26. Freitas, C., Effect of Light and Temperature on Zeta Potential and Physical Stability in Solid Lipid Nanoparticle (SLN $\left.{ }^{\mathrm{TM}}\right)$ Dispersions. Int. J. Pharm. (Amsterdam, Neth.) 1998. 168 (2), 221-229

27. Jiang, J.; Oberdörster, G.; Biswas, P., Characterization of Size, Surface Charge, and Agglomeration State of Nanoparticle Dispersions for Toxicological Studies. J. Nanopart. Res. 2009 , 11,77-89.

28. Griffith, A.; Notley, S., ph Dependent Stability of Aqueous Suspensions of Graphene with Adsorbed Weakly Ionisable Cationic Polyelectrolyte. J. Colloid Interface Sci. 2012, 369 (1), 210-215.

29. White, B.; Banerjee, S.; O'Brien, S.; Turro, N.; Herman, I., Zeta-Potential Measurements of Surfactant-Wrapped Individual Single-Walled Carbon Nanotubes. J. Phys. Chem. C 2007 111(37), 13684-13690.

30. Dasgupta, B. R.; Tee, S.-Y.; Crocker, J. C.; Frisken, B. J.; Weitz, D. A., Microrheology of Polyethylene Oxide Using Diffusing Wave Spectroscopy and Single Scattering. Phys. Rev. E: Stat. Phys., Plasmas, Fluids, Relat. Interdiscip. Top.2002, 65 (5), 051505.

31. Urry, D. W., What Sustains Life? Consilient Mechanisms for Pprotein-Based Machines and Materials. Springer-Verlag: New York, 2006. 
Insert Table of Contents artwork here

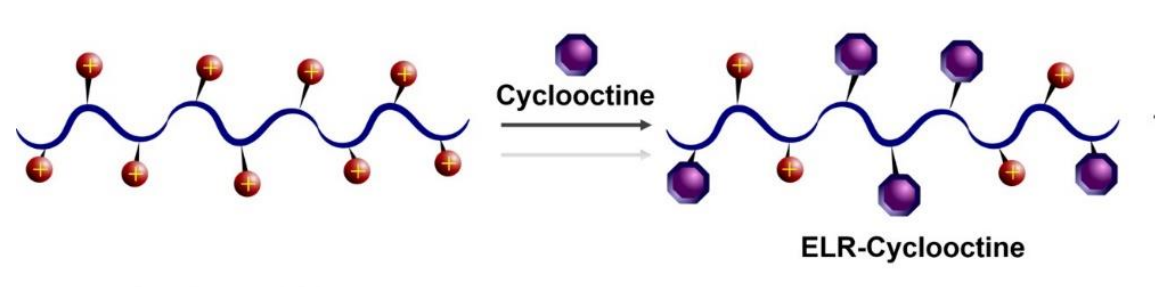

Poly lysine ELRs
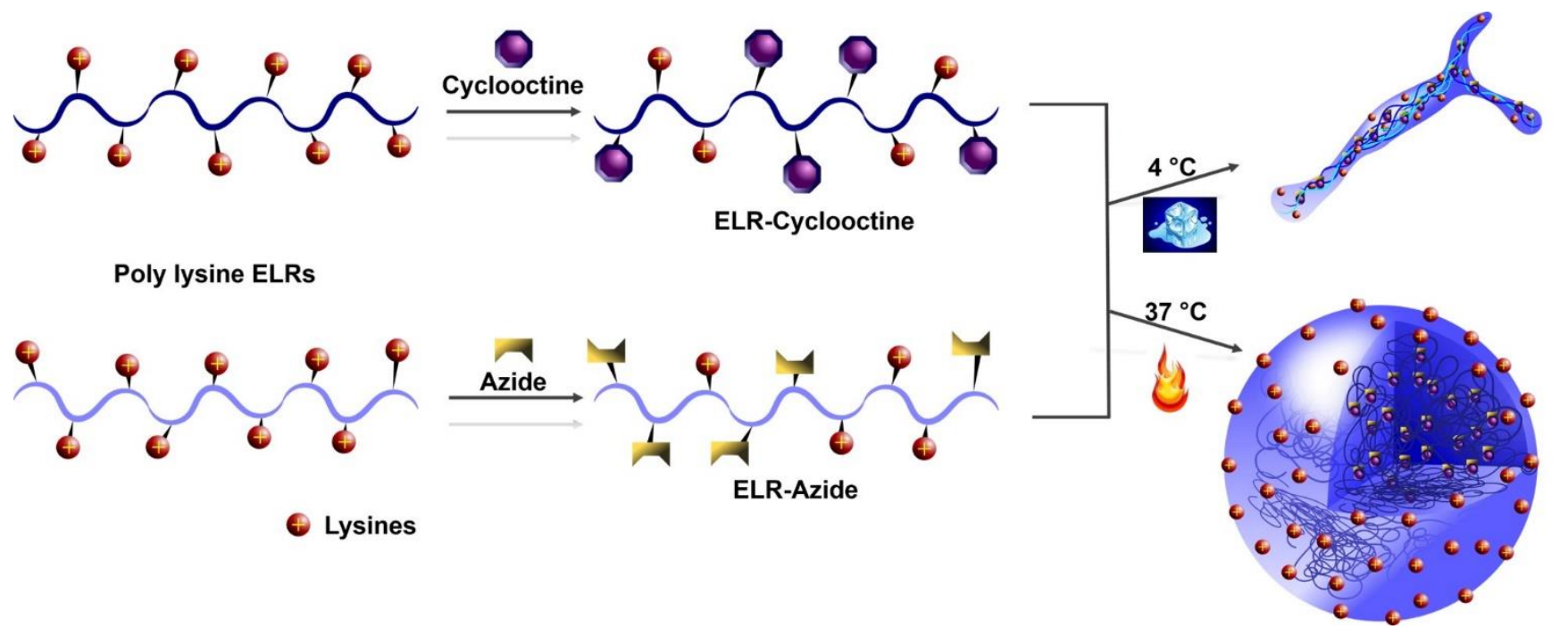